\title{
De la terre à la parenté dans le Haut Atlas marocain
}

Béatrice Lecestre-Rollier

\section{(2) OpenEdition}

Journals

Édition électronique

URL : https://journals.openedition.org/tc/1421

DOI : $10.4000 /$ tc. 1421

ISSN : 1952-420X

Éditeur

Éditions de l'EHESS

Édition imprimée

Date de publication : 1 avril 2003

ISSN : 0248-6016

\section{Référence électronique}

Béatrice Lecestre-Rollier, « De la terre à la parenté dans le Haut Atlas marocain », Techniques \& Culture [En ligne], 40 | 2003, mis en ligne le 18 mai 2006, consulté le 29 septembre 2022. URL : http:// journals.openedition.org/tc/1421; DOI : https://doi.org/10.4000/tc.1421

Ce document a été généré automatiquement le 29 septembre 2022.

Tous droits réservés 


\title{
De la terre à la parenté dans le Haut Atlas marocain
}

\author{
Béatrice Lecestre-Rollier
}

1 À partir d'une relecture de nos matériaux de terrain, relatifs aux sociétés berbérophones du Haut Atlas marocain, nous proposons de questionner l'hypothèse émise par certains auteurs d'une correspondance dialectique entre les différents niveaux de l'organisation sociale, voire d'une homologie entre processus techniques et processus sociaux. Il s'agit de repérer, à l'aide d'exemples, comment saisir concrètement les rapports milieu/technique/société ou culture. Précisons d'emblée que nous n'abordons guère l'étude des processus strictement techniques d'action sur la matière (gestes, outils, produits), c'est-à-dire les rapports entre l'homme et le matériel, mais que nous nous intéressons à l'ensemble des processus économiques qui ont trait à la production, selon une conception large du fait techno-économique.

Conditions techniques de la production et formes d'organisation familiale

2 Il existe dans notre zone d'étude deux types d'alliances matrimoniales: les unes fortement endogames, qui unissent des familles déjà proches, aussi bien géographiquement que parentalement -l'exemple le plus représentatif est celui du mariage "arabe » entre cousins parallèles patrilatéraux-, les autres au contraire fortement exogames, où les femmes sont mariées à l'extérieur du groupe montagnard, en plaine ou sur les piémonts. On peut proposer toutes sortes d'explications à ces modalités d'alliance. Mais si on ne repère pas que la carte des alliances en plaine se juxtapose parfaitement à celle des voies de la transhumance hivernale, on passe assurément à côté d'une explication fondamentale. Les montagnards ont absolument besoin de pouvoir accéder aux parcours pastoraux de plaine ou des piémonts l'hiver, quand la neige restreint ou empêche les déplacements en montagne. Car ils ne sont pas alors en mesure de subvenir au-delà de quelques jours aux besoins du cheptel ovincaprin, compte tenu des maigres ressources agricoles dont ils disposent et qui sont affectées prioritairement à l'alimentation humaine, ainsi qu'aux bovins et aux équidés. Par conséquent, établir des relations d'alliance avec des partenaires établis en plaine ou bien renforcer par des alliances des liens déjà existants s'avère une nécessité vitale 
pour les groupes montagnards, même si de telles relations ne sont mobilisées qu'occasionellement. En effet, il peut ne pas neiger durant tout un hiver ou même pendant plusieurs hivers successifs. Il peut également neiger peu abondamment; ou bien la couche de neige peut fondre rapidement, ce qui n'entrave guère au-delà de quelques jours les déplacements des animaux sur les parcours pastoraux. Cependant, s'il neige abondamment ou régulièrement, si le froid s'abat sur la montagne, alors les bêtes ne résistent pas longtemps aux rigueurs de l'hiver si elles n'ont pas la possibilité de quitter la montagne pour les piémonts ou la plaine.

Ainsi que nous l'avons montré dans un précédent article (Lecestre-Rollier \& GarriguesCresswell 2001), dans ce milieu marqué par les aléas, d'ordre climatique comme sociopolitique, la société s'organise par rapport aux périodes critiques, même si cellesci apparaissent, quantitativement, peu fréquentes. C'est moins par rapport aux périodes de plénitude qu'en fonction des risques de pénurie que se comprennent de nombreux traits de l'organisation sociale. Aussi l'analyse des rapports entre le milieu, les techniques et l'organisation socioculturelle ne peut-elle faire abstraction de cette donnée. Avant d'envisager des explications qui relèveraient d'une logique purement sociale, il importe d'épuiser la gamme des réponses suggérées par l'adaptation au milieu, compte tenu bien évidemment de l'état des techniques.

Que l'on ne se méprenne pas: il ne s'agit pas de raisonner en termes de déterminisme du milieu sur la société. En soi, le milieu ne dicte pas l'établissement de relations matrimoniales entre la montagne et la plaine. D'une part les rapports milieu/société sont médiatisés par les techniques: c'est parce que les techniques agro-pastorales ne permettent pas aux montagnards de nourrir le cheptel ovin-caprin sur les ressources agricoles produites en montagne que celui-ci est strictement dépendant des parcours pastoraux; et l'économie des hautes vallées ne permet pas davantage aux montagnards de se procurer à l'extérieur suffisamment de grain et de fourrage pour pallier l'absence momentanée des parcours. D'autre part, il existe bien des façons de répondre à la nécessité d'établir des liens avec la plaine. Si certains groupes privilégient la voie des alliances matrimoniales, d'autres, en revanche, ont choisi d'établir des alliances politiques; de même que certains groupes se tournent vers le nord tandis que d'autres se dirigent vers le sud et les piémonts qui bordent le Sahara. Aux contraintes imposées par le milieu physique et technique, les réponses du milieu social et culturel varient dans des limites qu'il s'agit de préciser. C'est, fondamentalement, au lieu même de cette articulation entre les données -environnementales, techniques et socioculturellesqu'il importe de réfléchir.

Un autre exemple de l'adaptation de l'organisation sociale au milieu montagnard nous est fourni par la façon dont le groupe familial s'agence. La forme idéale de celui-ci est la famille étendue avec le maintien des collatéraux ensemble, soit du vivant du père et sous son autorité, soit après sa mort. Car ainsi, le groupe familial est capable de s'investir dans une pluri-activité, source d'équilibre et de prospérité. En effet, si la pénurie affecte momentanément une activité productive ou une ressource particulière, il est en mesure de minimiser les risques en se repliant sur les autres sources de revenus. Seules cette diversité et cette complémentarité, qui reposent sur une division optimale des tâches entre les membres du groupe -le berger, le cultivateur, le travailleur salarié détaché de façon saisonnière ou permanente de la vallée à la recherche de revenus complémentaires en plaine, éventuellement le commerçant, le guide touristique, le soldat, enfin le chef de famille libéré des activités économiques de 
production et qui peut se consacrer aux activités publiques et politiques- assurent à celui-ci une relative sécurité. Et seuls les groupes familiaux qui parviennent à réaliser cet idéal de coopération sont à même de croître et de prospérer.

On ne saurait donc comprendre les formes d'organisation familiale si on ne les relie pas aux conditions techniques de la production. Conditions qui n'impliquent nullement une seule forme possible de composition de la famille. Les réponses sociales là encore varient, et l'on rencontre différents cas de figure. Le feu ou foyer (takat) recouvre des réalités variées et ses frontières sont extensibles. Des frères mariés peuvent choisir, après le décès de leur père, de continuer à vivre ensemble avec femmes et enfants dans la grande maison familiale, en conservant une dimension collective totale : propriété, exploitation, habitation et consommation sont alors communes. Mais ils peuvent préférer, tout en conservant une propriété indivise de la terre et du troupeau, vivre dans des maisons indépendantes et gérer chacun son propre budget : la propriété est commune, l'habitation et la consommation séparées, soit que l'exploitation demeure collective, soit qu'elle corresponde à un partage des tâches bien défini (par exemple, un des frères s'occupe exclusivement des travaux agricoles, l'autre du troupeau, tandis qu'un troisième se spécialise dans le commerce ou dans le travail salarié en plaine, etc.). Ils ont encore la possibilité de rompre l'indivision familiale, c'est-à-dire de se partager l'héritage de leur père tout en continuant à vivre sous le même toit, voire à partager leur consommation et même parfois à s'entraider pour continuer à mettre en valeur le patrimoine pourtant divisé. Nombre de foyers sont dans cette situation intermédiaire entre indivision et division. Car, si l'évolution contemporaine pousse incontestablement dans le sens de la famille restreinte, en revanche les contraintes du système productif penchent encore fortement en faveur de la famille étendue. Ce qui explique ces formes familiales intermédiaires où, tout en essayant de s'affranchir de la collectivité, on compte encore largement sur les bénéfices que l'on peut en retirer. Seuls les individus les plus dynamiques ou les plus novateurs, qui expérimentent d'autres façons de produire, parviennent à s'en affranchir.

Une correspondance dialectique entre les différents niveaux de la réalité

7 S'il n'y a pas lieu de rechercher des déterminismes univoques entre le technique et le social, c'est aussi parce qu'aucune donnée technique n'existe indépendamment des hommes. Tout fait techno-économique est en même temps -forcément- un fait social dans la mesure où il est pensé, vécu et manipulé par des hommes et des femmes insérés dans des sociétés. À l'inverse, tout fait social s'inscrit dans un certain contexte ou état technique et économique. Les faits sont donc dans la réalité indissociablement liés, tout autant des réalités matérielles que sociales et culturelles. On ne saurait, autrement qu'arbitrairement et pour des raisons heuristiques, isoler des données qui relèveraient d'un ordre matériel et d'autres qui relèveraient d'un ordre social.

Il est plus fructueux, selon Robert Cresswell, de tenter l'analyse des «réseaux dialectiques qui lient faber et sapiens" (1996: 14), c'est-à-dire l'homo faber manipulateur de la nature à l'homo sapiens penseur. Cet auteur suggère l'hypothèse d'une correspondance ou homologie dialectique entre les structures techniques et les structures socioculturelles. Il y a là l'idée qu'il pourrait exister un lien entre les processus techniques et les manifestations socioculturelles en général : ou bien, dans une société donnée, la forme particulière d'un processus technique est en rapport avec les formes de l'organisation sociale, ou alors, plus précisément, les processus techniques servent de véhicule pour les constructions symboliques de la société. 
Autrement dit, l'influence des systèmes techniques pourrait se révéler plus profonde qu'elle n'apparaît au premier abord; elle pourrait même atteindre les structures fondamentales de l'organisation sociale. Ce qui n'implique pas l'existence de relations de cause à effet, de lien mécanique entre les processus techniques et les formes de l'organisation sociale, mais suggère plutôt que quelque chose se retrouve, se répète entre ces deux ordres de faits qui nouent des rapports dialectiques :

«[...] la manière dont l'homme modèle la nature à son profit influant sur son comportement social et culturel et, réciproquement sa façon de concevoir et de régler ses rapports avec ses semblables informant parfois son choix de tel ou tel développement technique. » (Cresswell 1996 : 14).

Robert Cresswell propose aux anthropologues de tester cette hypothèse de travail, avec l'idée sous-jacente, plus vaste encore, que la configuration générale d'un réseau symbolisant un aspect de la vie sociale est homologue à la configuration générale de tous les autres réseaux symbolisant les autres aspects de la vie sociale et culturelle.

De l'enracinement à un territoire au lien familial

Dans le Haut Atlas, l'enracinement des groupes à un territoire se fonde sur la capacité de ceux-ci à s'investir dans l'exploitation et dans la défense de cet espace. Concrètement, pour le groupe familial, sur son aptitude à mettre en valeur un domaine, à l'investir physiquement, à le borner et à le défendre de la convoitise des autres groupes familiaux rivaux, voire à l'agrandir au détriment des leurs. Pour le groupe local, c'est parce qu'ils utilisent et défendent un territoire agro-sylvo-pastoral commun que les douars ou villages acquièrent et conservent des droits sur l'espace. Là aussi, ces droits sont susceptibles de se heurter à ceux des autres groupes locaux concurrents. Par exemple, les villages qui, démographiquement trop faibles, ne sont plus en mesure de maintenir une présence continue sur les hauteurs peuvent se voir déposséder de leurs droits par les villages voisins plus importants ou en expansion. Jadis, c'était en fonction de la capacité des groupes à prendre en charge l'ordre et la sécurité sur leurs terres que s'établissaient les droits de propriété des uns et des autres (Lecestre-Rollier 1997). Désormais, cela se fait au moyen d'épreuves judiciaires acharnées pour la jouissance des espaces collectifs, mais le processus reste le même : chaque localité doit s'efforcer de maintenir l'intégrité de son territoire, doit exercer son autorité sur cet espace et le protéger contre toute violation par un groupe étranger. De la même façon, au sein de la localité, chaque groupe familial, et au sein d'eux chaque homme, doit défendre ses propres espaces: ses terres, ses maisons et ses femmes. J'ai montré ailleurs (LecestreRollier 1999), suivant en cela les analyses fécondes de Jacques Berque, comment une grande partie du débat relatif au lien à la terre tourne dans ces sociétés autour de l'opposition entre le droit du sol et le droit de la mise en valeur. La reconduction infinie des litiges témoigne que ce débat n'est jamais achevé.

11 La terre est ici bien davantage qu'un simple capital économique. Elle ne suffit pas, d'ailleurs, à nourrir les hommes qui doivent s'efforcer de compléter les ressources qu'ils tirent de la montagne. Sa valeur est avant tout sociale : elle est enjeu d'identité et d'honneur. Autour d'elle se développent d'importantes stratégies qui visent à prendre position -ou à renforcer son pouvoir- au sein du groupe. Car c'est en possédant de la terre que l'on marque son appartenance au groupe, que l'on entre dans le jeu de l'honneur. Un homme n'est un homme d'honneur que s'il possède de la terre, la défend de la convoitise des autres et se lance lui-même dans la compétition pour s'accaparer leurs terres. On peut être riche de bien des façons, grâce au commerce ou au tourisme aujourd'hui, mais si l'on ne possède pas de terres, on ne saurait participer à la 
compétition pour l'honneur. Or seuls les hommes d'honneur sont responsables aux yeux de l'opinion, sont autorisés à prendre la parole lors des assemblées communautaires et sont à même de s'opposer aux autres dans les échanges. De la même façon, un groupe n'est un groupe d'honneur que s'il est capable de lancer des défis aux autres groupes, de relever les défis qu'on lui lance, donc de mettre en jeu son capital d'honneur. La terre est le lieu de l'échange entre les individus, les groupes familiaux et les groupes locaux.

On comprend dès lors pourquoi dans ces sociétés la territorialité est une référence prédominante, au point que la parenté même y est assujettie selon Raymond Jamous :

«La structure dominante chez les Iqar'iyen est celle du territoire. Dans ce cadre, la parenté ne constitue qu'une dimension englobée et dont toutes les caractéristiques sont assujetties au principe territorial [...]. On perd son identité agnatique si l'on quitte le territoire de son patrilignage » $(1981: 60)$.

13 La rupture du lien territorial entraîne celle du lien parental, comme l'intégration à un groupe parental protecteur s'impose là où on n'a pas de terre.

14 Le même principe directeur se retrouve quant à l'affiliation des individus au groupe de parenté d'une part, quant à l'intégration des groupes de parenté à la localité d'autre part. En effet, c'est parce qu'ils sont présents au sein du groupe ou de la localité, capables de s'investir dans des activités communes, que les individus sont reconnus comme appartenant à la communauté familiale et que les communautés familiales s'intègrent aux collectivités villageoises. Tout comme c'est en vertu de leur aptitude à s'investir dans l'exploitation et dans la défense d'un territoire que les individus et les groupes acquièrent et conservent des droits sur l'espace. La même structure organisatrice se dégage tant du côté de l'affiliation au groupe que du côté de la relation à la terre.

15 Au-delà d'une patrilinéarité et d'une patrilocalité idéalement proclamées, les pratiques vont dans le sens d'une très grande souplesse, faisant une large place à l'affiliation par les femmes (captation de gendres grâce à une alliance uxorilocale, captation des enfants des femmes qui reviennent dans leur patrilignage d'origine à la suite de leur divorce, recrutement de jeunes bergers qui grandissent au sein du groupe familial auquel ils finissent par s'intégrer définitivement en épousant une fille de la lignée, protection accordée autrefois aux exilés, etc.). La faible profondeur généalogique des lignées -à l'exception des lignées maraboutiques- entraîne leur vulnérabilité démographique. Aussi les pratiques visent avant toute chose à pérenniser le patrilignage en y intégrant des individus étrangers.

16 Une telle souplesse n'est possible qu'au prix de contraintes communautaires fortes. Les individus qui s'affilient de la sorte doivent s'effacer devant l'intérêt collectif et respecter le principe de solidarité qui fonde le groupe parental. Car, en l'absence de liens généalogiques, seule une coopération de tous les instants structure la famille et lui permet de prospérer. Cette solidarité se manifeste dans tous les domaines: dans le domaine économique grâce à la pluri-activité qui contribue à la cohésion du groupe, dans le domaine juridique où le principe de coresponsabilité lie les individus au groupe, dans le domaine politique grâce à l'activité du chef de famille qui rejaillit sur le prestige et l'honneur de tous. Tout cela contribue au bout du compte à créer des solidarités, à renforcer la dépendance des uns envers les autres, bref, à forger l'identité du groupe parental en l'absence de liens de filiation. Il s'agit de s'accepter comme parents puisque l'on partage une même destinée liée à la mise en valeur d'un patrimoine commun. 

d'une parenté commune, face à la diversité proclamée des origines, seule une solidarité collective forte institue la communauté locale. Le principe de responsabilité collective engendre l'appartenance au groupe; et la pérennité des intérêts partagés quant à l'occupation, la mise en valeur et la défense d'un territoire commun permet, outre l'organisation du système productif dans les meilleures conditions, de renforcer le sentiment d'unité. C'est dans le cadre de l'assemblée collective villageoise, qui se réunit chaque vendredi après la prière collective à la mosquée, que sont débattues les affaires publiques locales -et notamment tout ce qui concerne les principes de gestion des espaces agro-sylvo-pastoraux. logique du contrat, tant dans les rapports des hommes entre eux que dans les rapports des hommes à la terre. L'affiliation sociale et parentale est de même nature que l'affiliation au milieu. Ainsi que l'ont affirmé à maintes reprises des auteurs comme Maurice Godelier, André-Georges Haudricourt ou bien encore Jacques Barrau, les relations des hommes avec la nature sont indissociables des relations qu'entretiennent les hommes entre eux. C'est-à-dire que la façon dont les hommes s'approprient et gèrent la nature est comparable à la façon dont les individus et les familles se lient. Les mêmes principes, les mêmes agencements structurent les groupes et ancrent ceux-ci à un territoire. Les solidarités se forgent dans le cadre de la territorialité; l'ordre social se modèle sur l'ordre territorial; de la terre à la parenté se manifeste une certaine homologie de structure.

egique peut également se repérer à d'autres niveaux de l'organisation sociale, en particulier dans la vie rituelle (Garrigues-Cresswell 1997). Peut-on dès lors en déduire l'idée d'une homologie générale entre structures techno-économiques et structures socioculturelles ? Existe-t-il des homologies de réseaux entre les divers aspects de la vie sociale comme le suppose Robert Cresswell ?

ous semble approprié pour interroger l'idée de correspondance ou d'analogie entre les différents niveaux de l'organisation sociétale de renouer avec l'œuvre de M. Mauss. Nous le ferons à travers le beau livre que Camille Tarot (1999) a consacré à la relecture de cet auteur. Mauss, dans sa tentative de repenser une anthropologie jusque-là trop habituée à séparer les différents niveaux de la réalité, dans son refus sans cesse réaffirmé de réduire la totalité du social à certains domaines seulement, postule l'idée de rapports de traduction, c'est-à-dire de rapports d'expression, entre tous les niveaux du réel. Qu'il s'agisse de réconcilier l'individuel et le social, le psychologique, le biologique et le sociologique ou, au sein même de l'ordre sociologique, l'économique, le politique, le religieux, l'esthétique, etc., Camille Tarot montre bien comment ce concept de traduction se révèle central dans la pensée de Mauss. À l'encontre du concept de causalité qui implique l'idée mécanique de cause à effet, la notion de traduction renvoie à l'idée de circulation de sens entre tous les niveaux de la réalité qui se "traduisent " les uns dans les autres, qui s'habitent les uns les autres. Comme si le social était fait non pas de couches ou niveaux superposés, mais bien plutôt de multiples réseaux en connexion réciproque et dynamique. De sorte que du sens, de la force ne cessent de circuler de l'un à l'autre et ce dans toutes les directions: tout agit sur tout, tout interagit.

21 Le concept de traduction, en forçant la pensée à glisser d'un niveau à l'autre en tous sens, permet de prendre en compte la mobilité et le dynamisme des faits. Il permet de 
rompre avec le schéma trop simpliste d'une hiérarchie entre le haut et le bas, soit entre niveaux matériels et niveaux spirituels. Entendons-nous bien : il ne s'agit en aucun cas de nier l'importance du concept de causalité dans l'explication des rapports entre les faits, mais il importe de penser la complexité des faits au-delà d'une représentation mécanique de la causalité qui n'épuise pas leur interprétation. Peut-être est-ce une question d'échelle? Si la notion de causalité est opérante à une certaine échelle de relation entre les faits, au-delà elle apparaît beaucoup plus aléatoire. Tandis que la notion de traduction permet d'envisager une pensée de la complexité. Car tous les niveaux sont également signifiants et tous se présentent comme des totalités qu'il s'agit de traduire de l'un à l'autre.

La tâche de l'anthropologue est d'opérer cette traduction: d'abord, de parvenir à comprendre comment fonctionne un niveau (économique, politique, religieux ou autre) au sein d'une culture particulière, puis de traduire ce niveau dans un autre au sein de la même culture, enfin de relier les cultures entre elles en poursuivant toujours la même démarche de traduction. C'est à cette condition que le chercheur peut saisir les agencements d'idées, de valeurs, de normes, de signes qui forment la trame des représentations collectives. Non que se répètent tels quels d'un ordre à un autre les mêmes combinaisons d'éléments -toute traduction implique une certaine transformation dans un autre langage qui a ses particularités propres-, mais parce qu'il s'agit de repérer les structures profondes d'une culture, ces formes régulières qui se répètent dans les différentes pratiques sociales et qui sont comme des marqueurs culturels, des repères identitaires.

23 Une telle pensée de la complexité appelle l'idée de symbolisme. Le social est fondamentalement symbolique et, au sens maussien du terme, le symbolisme ne se réduit pas à un niveau parmi d'autres du social. Au contraire, il rend précisément compte du fait que le social est constitué de réseaux en connexion mutuelle. Camille Tarot nous le rappelle magistralement !

Nous pourrions également nous référer à la pensée de Claude Lévi-Strauss qui postule lui aussi l'idée d'une certaine homologie de structure. Mais là où Lévi-Strauss voit une structure d'ordre inconscient s'imposant aux différents niveaux de la construction du social, Mauss ne privilégie pas un domaine de l'organisation socioculturelle plus qu'un autre. Autant il nous semble que Lévi-Strauss, qui identifie le social au langage et le langage aux infrastructures mentales de son fonctionnement, perd ou saute des niveaux d'analyse, autant Mauss ne perd rien de la complexité des niveaux du réel, tous signifiants et tous à saisir en tant que totalités. Le concept maussien de fait social total renvoie bien à l'idée que chaque fait est lié aux autres, à tous les autres, ce qui explique que chaque croyance, chaque institution met en œuvre la totalité de la société sans pour autant représenter cette totalité à elle seule. Un fait social total engage toute la société; il traverse les découpages sociétaux en ce sens qu'il fait partie d'un système de représentation qui n'est pas uniquement lié au niveau particulier auquel il se rattache, mais qui est en rapport avec l'ensemble du système des représentations collectives. La nature même des faits, synthétique, impose cette idée d'imbrication des niveaux de la réalité où tout se tient et où tout agit sur tout, à l'image d'un réseau où tout est connecté à tout.

Il faut véritablement prendre acte de ce que cela signifie : non seulement repérer la façon et le lieu où s'établit la communication entre les faits -l'architecture du réseau en quelque sorte- mais aussi étudier toutes les implications de ces connexions 
multiples et pas uniquement celle entre langage et social, largement privilégiée dans la tradition française héritière de Lévi-Strauss. La liaison technique/social a eu de grands penseurs, tel André Leroi-Gourhan, dont il ne faudrait ni oublier ni minimiser l'héritage. Ce que Leroi-Gourhan appelait "le corps» ou «le squelette technoéconomique » des sociétés nous rappelle que toute société se construit, certes à partir d'êtres humains, certes à partir de mots, mais aussi à partir d'outils et l'on ne saurait faire l'impasse sur cette partie de notre programme de recherche.

\section{BIBLIOGRAPHIE}

Barrau, Jacques

1990. «Les hommes dans la nature. Esquisse d'une histoire naturelle des sociétés et des mœurs humaines ", Histoire des mœurs, T.1, Les coordonnées de l'homme et la culture matérielle, in J. Poirier (ed.). Paris : Gallimard (« La Pléiade »).

Berque, Jacques

1995. Structures sociales du Haut Atlas. Paris : Presses Universitaires de France.

Cresswell, Robert

1996. Prométhée ou Pandore? Propos de technologie culturelle. Paris : Éditions Kimé

(«Anthropologies »).

Garrigues-Cresswell, Martine

1997. «Permanence et modernité : la logique du cumul chez les sociétés berbères du Haut Atlas marocain ", pp. 42-57, in Jacques Berque. La Méditerranée, le Haut Atlas. Aix en provence :

Publications de l'Université de Provence.

Garrigues-Cresswell, Martine \& Béatrice Lecestre-Rollier

2001. « Gérer les aléas. Les sociétés du Haut Atlas marocain », Techniques \& Culture 38 : 69-95.

Godelier, Maurice

1984. L'Idéel et le matériel. Paris : Fayard.

Haudricourt, André-Georges

1987 La Technologie, science humaine. Recherches d'histoire et d'ethnologie des techniques. Paris :

Éditions de la Maison des Sciences de l'Homme.

Jamous, Raymond

1981. Honneur et baraka. Les structures sociales traditionnelles dans le Rif. Paris : Éditions de la Maison des Sciences de l'Homme.

Lecestre-Rollier, Béatrice

1997. «Identité et altérité : la logique du contrat dans les sociétés berbères du Haut Atlas

marocain ", pp. 19-41, in Jacques Berque. La Méditerranée, le Haut Atlas. Aix en provence :

Publications de l'Université de Provence.

1999. «La loi, le pouvoir et les acteurs : jeux et enjeux au Maroc. L'exemple d'un conflit foncier », pp. 295-331, in J.-L. Jamard, A. Montigny et F.-R. Picon (eds), Dans le sillage des techniques. Hommage à Robert Cresswell. Paris : L'Harmattan. 
Tarot, Camille

1999. De Durkheim à Mauss, l'invention du symbolique. Paris : La découverte (« Recherche », la

Bibliothèque du MAUSS).

\section{RÉSUMÉS}

Comment analyser les rapports entre les conditions techno-économiques de la production et les formes de l'organisation sociale ? À travers l'étude de quelques modalités de l'organisation parentale dans les sociétés du Haut Atlas marocain, l'auteur met en évidence la nécessité d'examiner la gamme des réponses suggérées par l'adaptation au milieu, compte tenu du contexte techno-économique, avant d'envisager des explications relevant d'une logique purement sociale. Mais plutôt que d'analyser en termes de causalité, même plurielle, les rapports des processus techniques et des processus sociaux, il semble fructueux d'envisager l'articulation sous la forme d'une homologie de réseaux entre les différents niveaux de la réalité, selon l'hypothèse proposée par Robert Cresswell.

From land to kinship in the Moroccan High Atlas.

How should one go about analyzing the techno-economic conditions of production and the form of social organization? Through a study of some patterns of kinship structure in the groups of the Moroccan High Atlas the author points up the need to completely cover the ranged of answers suggested by the necessity of adapting to the natural environment, taking into account the techno-economic context, before going on to explanations deriving from purely social logic. But rather than analyzing relations between technological and sociological processes in forms of simple or even multiple causes, it would seem more fruitful to conceive these relations as links between homologous networks on different levels of reality, according to the hypotheses proposed by Robert Cresswell.

\section{De la tierra al parentesco en el Alto Atlas marroquí.}

¿Cómo concebir las relaciones entre condiciones tecno-económicas de la producción y formas de la organización social ? A través del análisis de algunas modalidades de la organización parental en las sociedades del Alto Atlas marroquí, se hará evidente la necesidad de agotar la gama de respuestas sugeridas por la adaptación al medio, en función del contexto tecno-económico, antes de considerar explicaciones derivando de una lógica puramente social. Pero más que un análisis en términos de causalidad, incluso plural, de las relaciones entre procesos técnicos y procesos sociales, parece fructuoso el considerar su articulación bajo la forma de una homología de redes entre los distintos niveles de la realidad, según la hipótesis propuesta por Robert Cresswell.

\section{INDEX}

Mots-clés : Maroc, parenté, Haut-Atlas, organisation sociale

Keywords : Kinship, Hight Atlas, Marocco, social organization

\section{AUTEUR}

\section{BÉATRICE LECESTRE-ROLLIER}

Université René Descartes - Paris V/Techniques et culture, CNRS, 27 rue Paul Bert, 94204 Ivry Cedex. 